\title{
Tocotrienols reach the brain and play roles in the attenuation of body weight gain and improvement of cognitive function in high-fat diet-treated mice
}

\author{
Yugo Kato, ${ }^{1}$ Hayami Uchiumi, ${ }^{2}$ Ryo Usami, ${ }^{2}$ Hirokatsu Takatsu, ${ }^{3}$ Yoshinori Aoki, ${ }^{4}$ Shuichi Yanai, ${ }^{5}$ Shogo Endo, ${ }^{5}$ and \\ Koji Fukui ${ }^{1,2, *}$ \\ 'Molecular Cell Biology Laboratory, Department of Functional Control Systems, Graduate School of Engineering and Science \\ and ${ }^{2}$ Molecular Cell Biology Laboratory, Department of Bioscience and Engineering, College of Systems Engineering and Science, \\ Shibaura Institute of Technology, Fukasaku 307, Minuma-ku, Saitama 337-8570, Japan \\ ${ }^{3}$ Department of Medical Technology, Faculty of Health Sciences, Kyorin University, Shimorenjaku 5-4-1, Mitaka, Tokyo 181-8612, Japan \\ ${ }^{4}$ Mitsubishi-Chemical Foods Corporation, Marunouchi 1-1-1, Chiyoda-ku, Tokyo 100-8251, Japan \\ ${ }^{5}$ Aging Neuroscience Research Team, Tokyo Metropolitan Institute of Gerontology, Sakae-cho 35-2, Itabashi-ku, Tokyo 173-0015, Japan
}

(Received 19 January, 2021; Accepted 30 March, 2021)

\begin{abstract}
Obesity induces severe disorders such as type 2 diabetes and cardiovascular events, and the number of people with obesity is increasing all over the world. Furthermore, it is possible that obesity increases the risk of cognitive dysfunction via the acceleration of oxidative damage. Tocotrienols, which are part of the vitamin E family, have antioxidant and anti-obesity effects. However, the effects of tocotrienols on high-fat diet-treated mice have not been completely elucidated. In this study, we assessed changes in body weight, spatial reference memory acquisition, liver lipid droplet size, blood brain barrier-related protein expressions and antioxidative defense systems in high-fat diet-treated mice in the presence or absence of tocotrienols. The results showed that tocotrienols significantly inhibited body weight gain and lipid droplet synthesis. Although the amount was very small, it was confirmed that tocotrienols surely reached the brain in the perfused brain. Treatment with tocotrienols was tended to improve cognitive function in the control mice. However, tocotrienols did not modulate blood brain barrierrelated protein expressions or antioxidative defense systems. These results indicate that treatment with tocotrienols could be effective for the prevention of obesity and cognitive dysfunction. Further extended research is needed to elucidate the relationship between anti-obesity and antioxidant effects of tocotrienols, especially in the brain.
\end{abstract}

Key Words: vitamin E, anti-obesity effects, blood brain barrier, antioxidative defense systems, fatty liver

$\mathrm{O}$ besity is a major medical problem all over the world and causes many severe secondary diseases such as type 2 diabetes and cardiovascular disease. ${ }^{(1,2)}$ Because these diseases increase medical costs and the caregiver burden, public attention is focused on obesity prevention. One possible mechanism of obesity is oxidative damage by reactive oxygen species (ROS). ${ }^{(3)}$ The ROS production and the accumulation of oxidative damage associated with obesity are deeply involved in the onset of many diseases such as type 2 diabetes, cardiovascular disease, and nonalcoholic steathepatitis. ${ }^{(4-6)}$ Oxidative damage in the brain has been shown to attenuate cognitive function in rodent models. ${ }^{(7,8)}$ Numerous studies have reported a relationship between obesity and an increased risk of Alzheimer's disease (AD). ${ }^{(9,10)}$ However, the relationship between oxidation and cognitive dysfunction in obesity has not been elucidated in detail.

Several lines of evidence have been demonstrated that natural compounds such as caffeine and ginger have strong anti-obesity effects. ${ }^{(11,12)}$ We are also interested in the anti-obesity effects of natural compounds, and have therefore been focusing on tocotrienols (T3s), which are part of the vitamin E (VE) family.

VE is classified into tocopherols (TOCs) and T3s based on the presence or absence of double bonds on the phytyl side chain, respectively. There are four isoforms $(\alpha-, \beta-, \gamma-$, and $\delta$-) in TOCs and $\mathrm{T} 3 \mathrm{~s}$ according to differences in the number and position of the methyl group on the chroman ring. ${ }^{(13)}$ It is well known that VE exhibits strong antioxidant function both in vivo and in vitro, ${ }^{(14,15)}$ and T3s have specific beneficial functions not found in TOCs. For example, T3s have neuroprotective, anti-obesity and anti-cancer effects. ${ }^{(16-18)}$ However, a few contradictions were found in an anti-obesity studies on T3s. ${ }^{(17,19)}$ As for the reasons for the contradictory results regarding the anti-obesity effects of T3s, we think that the experimental conditions (e.g., type of diet, compositional balance of T3s isoforms, treatment period, volume of $\mathrm{T} 3 \mathrm{~s}$ ) differed between each research group. We previously reported that $\mathrm{T} 3 \mathrm{~s}$ significantly inhibited body weight gain in high-fat diet (HFD)-treated mice. ${ }^{(20,21)}$ Although we recognized the anti-obesity effects of T3s, no change was seen in antioxidative enzyme activities or cognitive and motor functions using several maze apparatuses. On the other hands, other our previous study demonstrated that T3s prevented cognitive dysfunction by attenuating oxidative stress in the rat brain. ${ }^{(14)} \mathrm{T} 3 \mathrm{~s}$ need to cross the blood brain barrier (BBB) to exert a neuroprotective effect; however, some reports have indicated that $\mathrm{T} 3 \mathrm{~s}$ cannot cross the $\mathrm{BBB}^{(14)}$ which regulates the exchange of substances between capillary blood vessels and neurons in the brain. ${ }^{(22)}$ It is well known that tight junction is a complex of BBB system, and is composed of the specific proteins such as Occludin, Claudin 5 , and Junctional adhesion molecule (JAM) 1. ${ }^{(23-25)}$ These tight junction-related proteins are injured by oxidative damage and during aging. As a result, neurons are more likely to be attacked by oxidative stress and other factors. However, to our knowledge, no evidence of obesity-induced oxidative damage in the brain or

*To whom correspondence should be addressed.

E-mail: koji@sic.shibaura-it.ac.jp

He received "SFRR Japan Award of Scientific Excellence" in 2020 in recognition of his outstanding work. 
the neuroprotective effects of T3s has been presented.

Therefore, this study aimed to assess the beneficial effects of T3s excluding body weight gain, to confirm whether T3s reach the brain, and to assess the changes in cognitive function and antioxidative defense systems in the brain. To achieve these aims, we measured the cognitive and motor functions of mice using three apparatuses (the Morris water maze, Rota-Rod, and Ymaze) and assessed antioxidative defense systems in the brain. Additionally, we checked T3s levels and BBB-related protein expressions in the brain after perfusion.

\section{Materials and Methods}

Animals. All animal experiments were approved by the Animal Protection and Ethics Committee of Shibaura Institute of Technology, Tokyo, Japan (Approval No.: 19002). C57BL/6 Ncr male mice were purchased from Japan SLC, Inc. (Hamamatsu, Japan) and housed under controlled conditions $\left(24 \pm 2{ }^{\circ} \mathrm{C}, 12 \mathrm{~h}\right.$ light/dark cycle). All mice had ad libitum access to food and water. Mice $(n=40)$ were fed a Control diet (Ctrl; \#D12450, Research Diets Inc., New Brunswick, NJ) or a HFD (\#D12492, Research Diets Inc.) in the presence or absence of $\mathrm{T} 3 \mathrm{~s}$ (Mitsubishi-Chemical Foods Corp., Tokyo, Japan) $(50 \mathrm{mg} / 100 \mathrm{~g}$ diet) for 8 weeks from 4 weeks of age (Ctrl with T3s: Ctrl + T3s, HFD with T3s: HFD + T3s). The nutrient composition of each diet is described in our previous paper. ${ }^{(21)}$ Ten mice were assigned to each group ( $n=10$ in each group). Body weight was measured once a week in all mice. After the treatment period, cognitive and motor functions were assessed, as described below. The mice were then euthanized to collect liver and brain tissues for each measurement. Four mice from each group were perfused with phosphate-buffered saline (PBS) to remove blood from the tissues. All other chemical reagents were obtained from FUJIFILM Wako Pure Chemical Corporation (Osaka, Japan).

Measurement of cognitive function. The Morris water maze test was used to measure spatial memory ability, as described previously with some modifications. ${ }^{(21)}$ The mice swam in the apparatus (Muromachi Kikai Co., Ltd., Tokyo, Japan; 140 $\mathrm{cm}$ in diameter, $45 \mathrm{~cm}$ in height). Before the cognitive trials, pretrials and handling were performed for 3 consecutive days. The mice were handled twice a day for $3 \mathrm{~min}$ by an experimenter, and swam freely for $3 \mathrm{~min}$ in the absence of a goal. The cognitive trials were performed for 5 consecutive days. The goal time (escape latency), swimming time ratio in the platform quadrant and the average swimming speed were analyzed using ANYmaze software (ver. 4.98; Stoelting Co., Wood Dale, IL). The learning ratio was then calculated (the ratio of Day $\mathrm{X}$ was calculated as the goal time on day $\mathrm{X}$ divided by the goal time on day 1 was subtracted from 1 and multiplied by 100). All abnormal values were rejected based on the goal time on the final test day. If the average goal time on the final day was over $135 \mathrm{~s}$, the data for that mouse were rejected.

Coordinated movement was measured using Rota-Rod (Muromachi Kikai Co., Ltd.), as described previously with some modifications. ${ }^{(21)}$ The time when the mice fell from the rod and the rpm at the fall were measured. Three times per day (every $20 \mathrm{~min}$ ).

The short-term memory of each mouse was measured using the Y-maze apparatus (arm length: $425 \mathrm{~mm}$, width: $40 \mathrm{~mm}$, height: $100 \mathrm{~mm}$, angle between each pair of arms: $120^{\circ}$; Muromachi Kikai Co., Ltd.). All mice started from the same arm and moved freely for $10 \mathrm{~min}$. The arm entering times were analyzed using ANY-maze software. The alternation score was calculated by measuring the number of times the mouse entered different arms in succession.

Histological analysis. Liver tissues, which were perfused, were fixed by paraformaldehyde, dehydrated by sucrose solution, and embedded in O.C.T. compound (Sakura Finetek UAS Inc.,
Torrance, CA). After slicing, the samples were plated on a slide glass (Matsunami Glass Ind., Ltd., Osaka, Japan) and subjected to hematoxylin \& eosin (HE) and Oil Red O staining. ImageJ software (National Institutes of Health, Bethesda, MD) was used for analysis of Oil Red O.

Measurement of VE levels. All VE isoforms were measured by the saponification extraction method, as described previously, with some modifications. ${ }^{(21)} 10 \%$ homogenates of liver and each brain region were prepared using PBS. The sample homogenates, $35 \%$ potassium hydroxide, and $6 \%$ pyrogallol solution were mixed, and then all samples were saponified at $100^{\circ} \mathrm{C}$ for $45 \mathrm{~min}$. 2,2,5,7,8-pentamethyl 6-chromanol (PMC) (Sigma Aldrich Corp., St. Louis, MO) was used as an internal standard. After $45 \mathrm{~min}$, the saturated saline solution and the mixture of hexane and ethyl acetate $(9: 1$, by volume) were added to all samples. After mixing, all samples were centrifugated at $3,000 \mathrm{rpm}$ and $4^{\circ} \mathrm{C}$ for $10 \mathrm{~min}$. After centrifugation, the upper layers were collected and washed by water and the saturated saline solution. The extracts were evaporated under nitrogen gas. The residues were dissolved by methanol. This solution was used for VE measurement. High performance liquid chromatography (HPLC) with electrochemical detection (Shiseido Co., Ltd., Tokyo, Japan) was used for the VE analysis. The mobile phase was composed of $\mathrm{H}_{2} \mathrm{O}_{2}$ and HPLC-grade methanol (3:97) including $50 \mathrm{mM}$ of $\mathrm{NaClO}_{4} \cdot \mathrm{H}_{2} \mathrm{O}$. The HPLC column [Develosil C30-UG-3 $(2.0 \times 250 \mathrm{~mm}$; Nomura Chemical Co., Ltd., Aichi, Japan)] was used at $5^{\circ} \mathrm{C}$.

Western blotting. Western blotting was performed as described previously, with some modifications. ${ }^{(21)}$ Briefly, twenty $\mu \mathrm{g}$ of protein in the samples was separated on a $7 \%, 12 \%$ or $15 \%$ sodium lauryl sulfate-polyacrylamide gels. The proteins were transferred to nitrocellulose membranes and then stained using Ponceau S solution (Sigma Aldrich Corp.). After staining, the membranes were incubated in blocking solution $[2 \%$ skim milk in Tris-HCl buffered saline (TBS) with Tween 20 (TBS-T)] for $60 \mathrm{~min}$. After blocking, the membranes were treated with primary antibodies overnight at $4^{\circ} \mathrm{C}$. Primary antibodies [antiOccludin antibody \#ab168986; Abcam Plc., Cambridge, UK, at 1:500 dilution, anti-Claudin 5 antibody \#ab15106; Abcam Plc. at 1:4,000, JAM1 polyclonal antibody \#bs-3651R; Bioss Inc., Wobun, MA, at 1:650, anti-Superoxide dismutase (SOD)1 antibody \#ab13498; Abcam Plc. at 1:2,500, anti-Glutathione peroxidase (GPx)1 antibody \#ab22604; Abcam Plc. at 1:4,000, antiCatalase (CAT) antibody \#ab195306; Abcam Plc. at 1:2,000, mammalian target of rapamycin (mTOR) antibody \#2972; Cell Signaling Technology (CST) Inc., Danvers, MA, at 1:1,000, phospho-mTOR (Ser2448) antibody \#2971; CST Inc. at 1:500, protein kinase B (Akt) \#9272; CST Inc. at 1:4,000, phospho-Akt (Ser473) antibody \#4060; CST Inc. at 1:1,000, or anti- $\alpha$-tubulin antibody \#2125; CST Inc. at 1:4,000]. Anti-rabbit IgG HRP antibody (Promega Corp., Madison, WI) was treated at 1:4,000 dilution. After washing the membranes, the chemiluminescent signals were detected using a luminescent image analyzer (LAS-3000; FUJIFILM Corporation, Tokyo, Japan). The relative intensities of all images were measured using ImageJ (National Institutes of Health).

Measurement of antioxidative enzyme activities. The activity of SOD was measured using the SOD Assay kit-WST (\#S311; Dojindo Laboratory, Kumamoto, Japan) according to the manufacturer's protocol. The absorbances were measured using a microplate reader (\#51119300, Multiskan GO; Thermo Fisher Scientific Inc., Hercules, CA).

The measurement of GPx activity was performed using a commercial kit (Glutathione peroxidase cellular activity assay kit; Sigma-Aldrich) according to the manufacturer's protocol.

CAT activity was calculated by monitoring hydrogen peroxide levels, as described previously with some modifications. ${ }^{(21)}$ The brain homogenate, K-phosphate buffer and hydrogen peroxide 
were mixed in 96-well plates. Finally, the absorbance at $240 \mathrm{~nm}$ was measured using a microplate reader (Multiskan GO, Thermo Fosher Scientific, Inc.).

Statistical analysis. Data are presented as the mean $\pm \mathrm{SE}$, and were analyzed using JMP 13.0 software (SAS Institute Inc., Cary, NC). For all data, the Tukey Kramer test was performed. Values of $p<0.05$ were considered to be statistically significant. The reason that the sample numbers differ between each group in the measurement of antioxidative enzyme activities, is that the sample volume in the hippocampus is very small.

\section{Results}

T3s significantly inhibited body weight gain in HFDtreated mice. Body weight was measured once a week for 8 consecutive weeks. The ratio of body weight gain in both HFDtreated groups was significantly increased 1 week after treatment compared to both control diet groups (Fig. 1). Additionally, cotreatment with HFD and T3s significantly inhibited body weight gain in the final 2 weeks compared to the HFD group. On the other hand, no significant difference was found between the Ctrl and $\mathrm{Ctrl}+\mathrm{T} 3$ s groups.

Cognitive function of each mouse by the Morris water maze test. Obesity has been reported to increase the risk of cognitive dysfunction such as AD. ${ }^{(9,10)}$ In this study, HFD significantly induced body weight gain, and T3s significantly attenuated body weight gain. Spatial memory ability was measured using the Morris water maze test (Fig. 2). The learning ratio of the Ctrl $+\mathrm{T} 3 \mathrm{~s}$ group was significantly higher than that of the Ctrl and HFD + T3s groups on the second trial day; however, no significant difference in the learning ratio was seen on the other test days.

To consider the difference of motor function, the average swimming speed was measured. No significant difference was observed in the presence or absence of T3s. Treatment with HFD significantly decreased the average swimming speed on the first test day regardless of the presence of T3s.

Cognitive function of each mouse by Y-maze test. The short-term memory of each mouse was measured using the Ymaze test (Fig. 3). No significant difference in short-term memory was observed; however, treatment with T3s nominally increased (but not significantly) the alternation scores in both diet groups. Additionally, treatment with HFD nominally decreased (but not significantly) the number of total arm entries regardless of the presence of T3s.

Cognitive function of each mouse by the Rota-Rod test. The coordinated movement function of each mouse was measured using the Rota-Rod test as an index of cognitive function (Fig. 4). Treatment with HFD significantly decreased the time to fall and rpm at the fall regardless of the presence of T3s.
No significant difference was found between the T3s-treated and untreated groups.

T3s inhibited accumulation of lipid droplets in liver tissue. T3s significantly inhibited body weight gain in the HFD-treated mice. The existence of lipid droplets in liver were checked by Oil Red O staining (Fig. 5). Treatment with HFD increased the number and size of lipid droplets compared to the Ctrl group. On the other hand, treatment with T3s showed small droplets compared to the untreated groups in HFD-treated mouse liver. Additionally, co-treatment with $\mathrm{T} 3 \mathrm{~s}$ in the control diet decreased the number of lipid droplets compared to the untreated group.

Oral intake of T3s significantly increased T3s levels in each tissue. Although we have measured T3s levels many times using HPLC, these samples were not perfused, and may have included a small amount of blood in the tissues. ${ }^{(20,21)}$ To examine the existence of T3s on the neurons, the tissues were collected after removing blood by perfusion, and T3s levels were measured. The results confirmed the existence of T3s (Fig. 6).

The results also showed that $\alpha$-T3 levels in the liver, cortex, and hippocampus of mice in the HFD + T3s group were significantly increased compared to those in the HFD group. Additionally, $\alpha-$ T3 levels in the liver and hippocampus in the HFD + T3s group were significantly higher than those in the $\mathrm{Ctrl}+\mathrm{T} 3 \mathrm{~s}$ group. Treatment with T3s significantly increased $\alpha$-T3 levels in the cortex and hippocampus compared to the untreated Ctrl groups. On the other hand, $\gamma$-T3 levels were increased only in the liver of mice in the HFD + T3s group.

BBB-related protein expressions showed no change in HFD-treated mice brain. In the former experiment, T3s were detected in the brain by HPLC analysis. However, some reports have indicated that T3s cannot cross the BBB. ${ }^{(14)}$ To clarify the relationship between brain $\mathrm{T} 3 \mathrm{~s}$ levels and $\mathrm{BBB}$ conditions, the expressions of tight junction-related proteins (Occludin, Claudin5, and JAM1) were measured by WB (Fig. 7). There were no significant differences of all proteins. However, treatment with $\mathrm{T} 3 \mathrm{~s}$ tended to increase expressions compared to the untreated groups.

Changes in antioxidative enzyme activities in each mouse brain. Significant differences were observed in the learning rate of T3s-treated mice in the Morris water maze test. It is well known that brain redox balance is very important for the maintenance of cognitive function, ${ }^{(7,8,14)}$ so some antioxidative enzyme activities were measured in the cerebral cortex and hippocampus of each mouse (Fig. 8). There was no significant difference in all parameters.

Changes in antioxidative enzyme expressions in each mouse brain. The expressions of antioxidative enzyme proteins were measured by WB (Fig. 9). No significant differences were observed for any enzymes. However, treatment with T3s nomi-

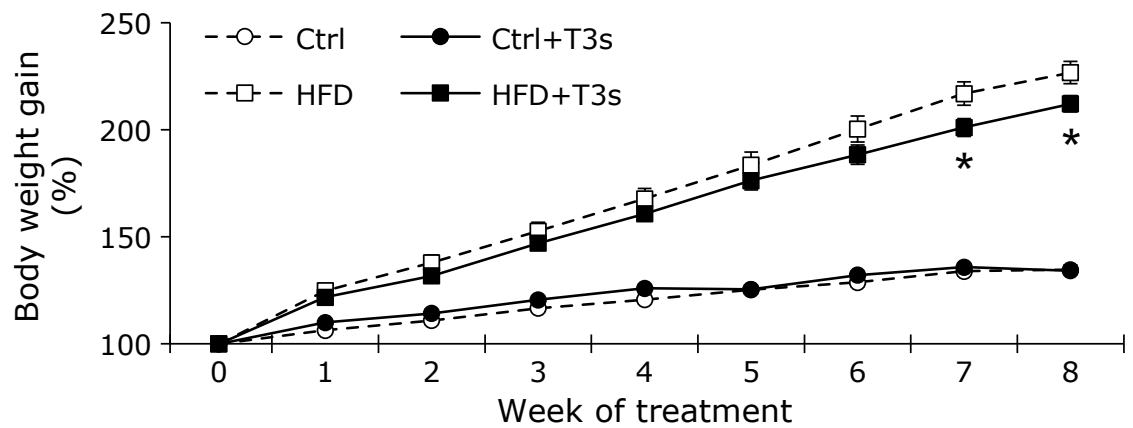

Fig. 1. Changes in body weight gain in each group. Body weight was measured once a week for 8 consecutive weeks. The ratio of body weight gain was calculated from the body weight of each mouse. The body weight at the starting week was set to $100 \%$. Control (Ctrl): $n=10, \mathrm{Ctrl}+\mathrm{T} 3 \mathrm{~s}$ : $n=10$, high-fat diet (HFD): $n=10$, HFD + T3s: $n=10$. The date was plotted as mean \pm SE. Tukey Kramer method: $0.01<p<0.05 *$, HFD vs HFD + T3s. 

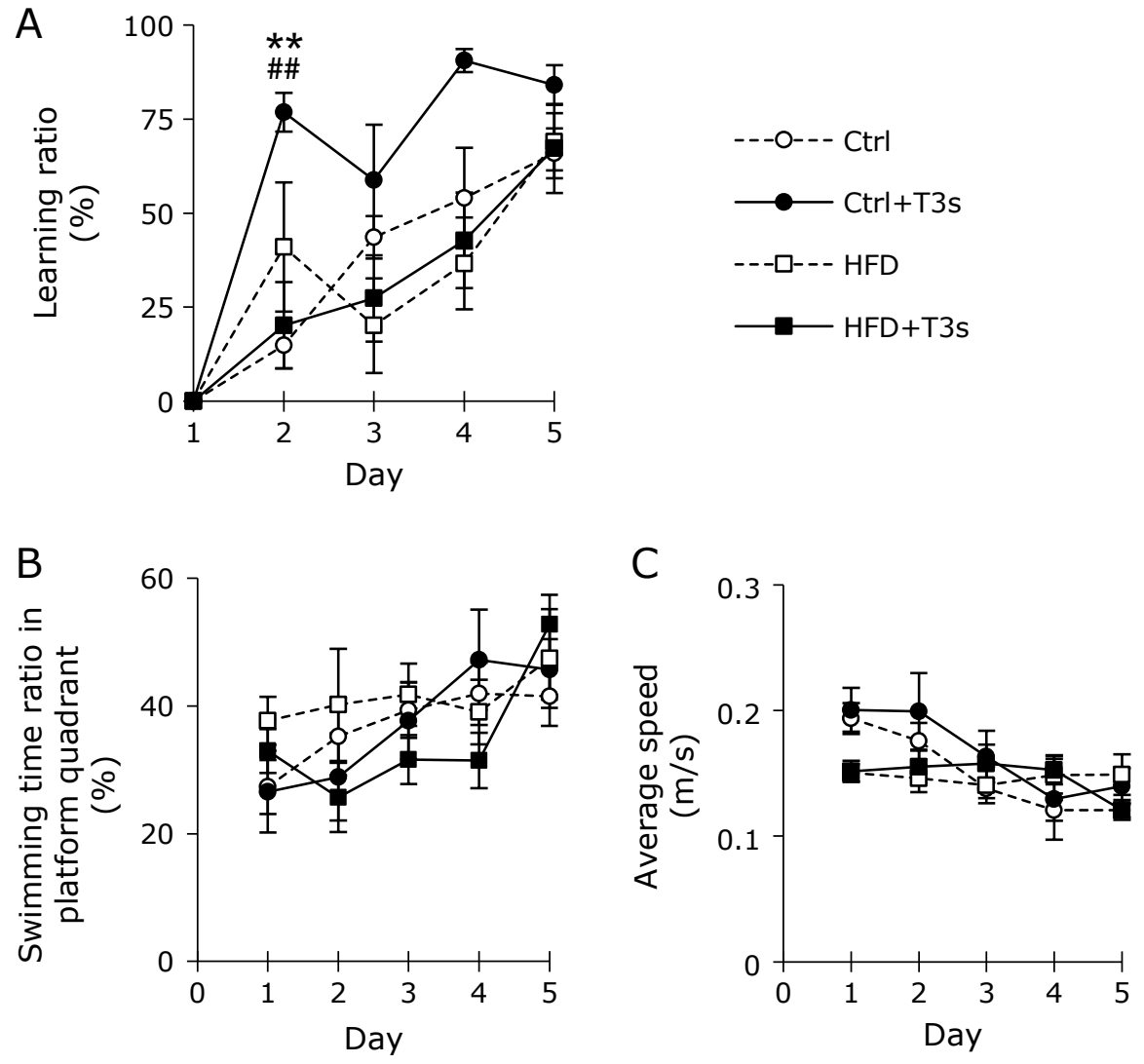

Fig. 2. The results of the Morris water maze test. Learning ratio (A), swimming time ratio in the platform quadrant (B), and average speed (C) were measured. The learning ratio was calculated from the escape latency. Control (Ctrl): $n=7, \mathrm{Ctrl}+\mathrm{T} 3 \mathrm{~s}: n=5$, high-fat diet (HFD): $n=5$, HFD + T3s: $n=7$. The date was plotted as mean \pm SE. Tukey Kramer method: $p<0.01 * * C$ trl vs Ctrl + T3s, $p<0.01^{\# \# ~ C t r l ~}+$ T3s vs HFD + T3s, $0.01<p<0.05^{\$} C t r l$ vs HFD, $0.01<p<0.05^{\dagger} \mathrm{Ctrl}+\mathrm{T} 3 \mathrm{~s}$ vs HFD + T3s.
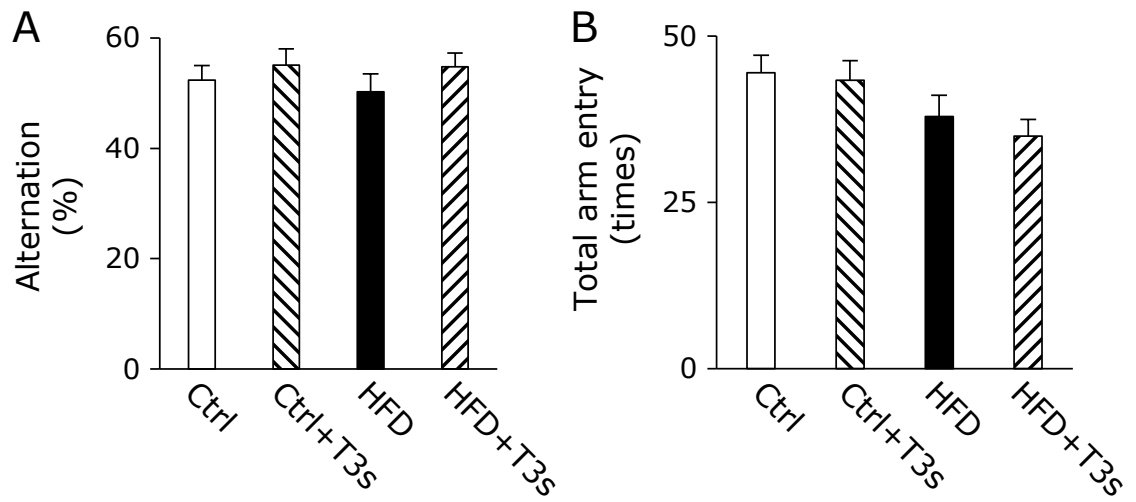

Fig. 3. Alternation scores (A) and total arm entries (B) were measured using the Y-maze test. Control (Ctrl): $n=10, \mathrm{Ctrl}+\mathrm{T} 3 \mathrm{~s}: n=10$, high-fat diet (HFD): $n=10$, HFD + T3s: $n=10$. The date was shown as mean \pm SE. Comparisons were performed using the Tukey Kramer method.

nally (but not significantly) increased protein expressions.

No change in the phosphorylation ratio of Akt and mTOR. It has been reported that the phosphorylation ratio of Akt and mTOR are modified by calorie restriction models. ${ }^{(26,27)}$ Additionally, these phosphorylation levels are involved in redox balance regulation. We checked whether treatment with HFD and/or T3s changed phosphorylation levels (Fig. 10). There were no significant differences in both phosphorylation levels. However, treatment with HFD and T3s tended to increase the phosphorylation ratio of both proteins.

\section{Discussion}

Recently, as the number of people with obesity has been increasing around the world, the anti-obesity effects of T3s have attracted increasing attention. However, the detailed mechanisms underlying the effects of T3s have yet to be elucidated. In this study, we investigated body weight gain in the presence or absence of T3s. Treatment with T3s significantly inhibited body weight gain in the HFD-treated group compared to the untreated. This inhibitory effect of T3s was confirmed in the final 2 weeks 

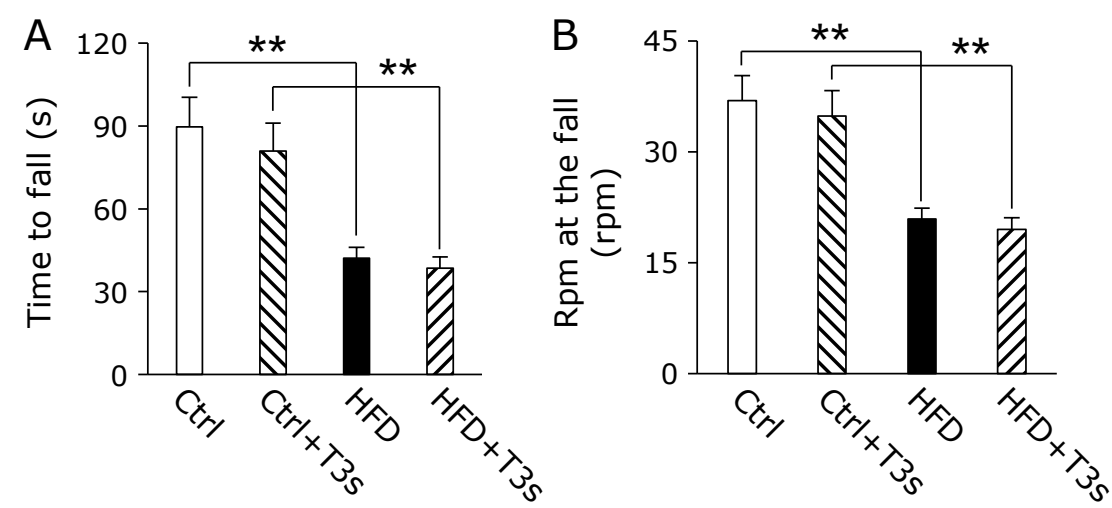

Fig. 4. The coordinated movement function of each mouse. The time to fall (A) and rpm at the fall (B) were measured using the Rota-Rod test. Control (Ctrl): $n=10, \mathrm{Ctrl}+$ T3s: $n=10$, high-fat diet (HFD): $n=10$, HFD + T3s: $n=10$. The date was shown as mean \pm SE. Tukey Kramer method: $* * p<0.01$

A

Ctrl

Ctrl+T3s

HFD

HFD+T3s
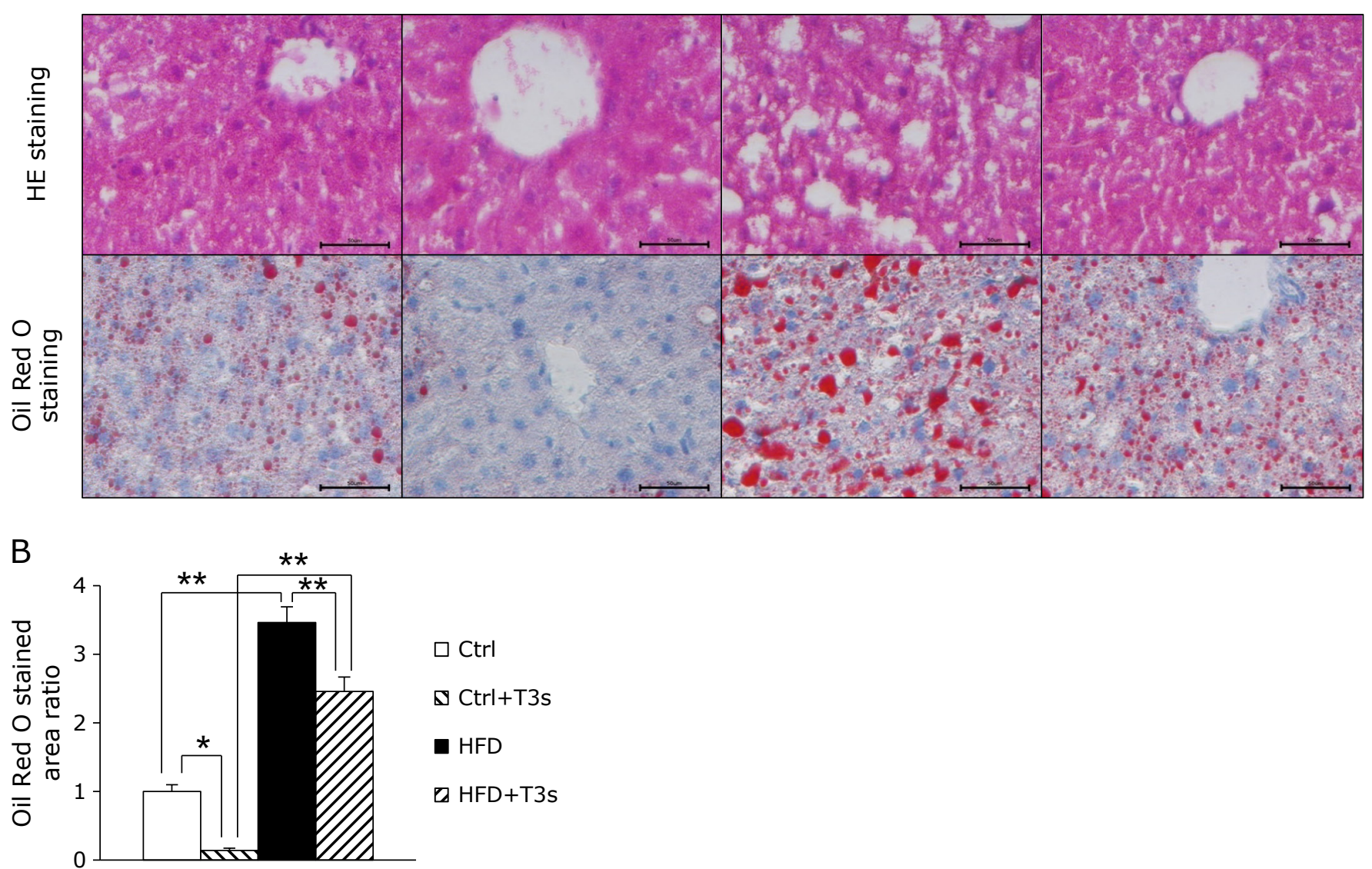

Fig. 5. Lipid droplets in the liver tissue of each mouse. Hematoxylin and eosin (HE) and Oil Red O staining were performed (A) and the stained area ratio by Oil Red O (B). Cytoplasm and nucleus were stained by HE staining, and lipid droplets were stained by Oil Red O staining. The scale bar $=50 \mu \mathrm{m}$. Control (Ctrl): $n=4, \mathrm{Ctrl}+\mathrm{T} 3 \mathrm{~s}: n=4$, high-fat diet (HFD): $n=4$, HFD + T3s: $n=4$. The date was shown as mean \pm SE. Tukey Kramer method: ${ }^{*} p<0.05,{ }^{* *} p<0.01$.

of the treatment period. Previously, we have already reported that long-term (5 months) treatment with T3s inhibited body weight gain in only the first half of the treatment period. ${ }^{(21)}$ These results indicated that T3s do not stop, but rather delay body weight gain. To clarify the beneficial effects of T3s excluding the attenuation of body weight gain in mice, we checked for the presence of lipid droplets in mouse liver. The size and number of lipid droplets were reduced in both T3s-treated groups. Lipid droplets contain triglyceride and cholesterol, and these parameters are increased in obesity. ${ }^{(28,29)}$ It has been reported that $\gamma$-T3 reduced the amount of triglyceride in Hepa 1-6 cells derived from a BW7756 tumor in a C57L mouse by modulating lipid synthesis related-mRNA 

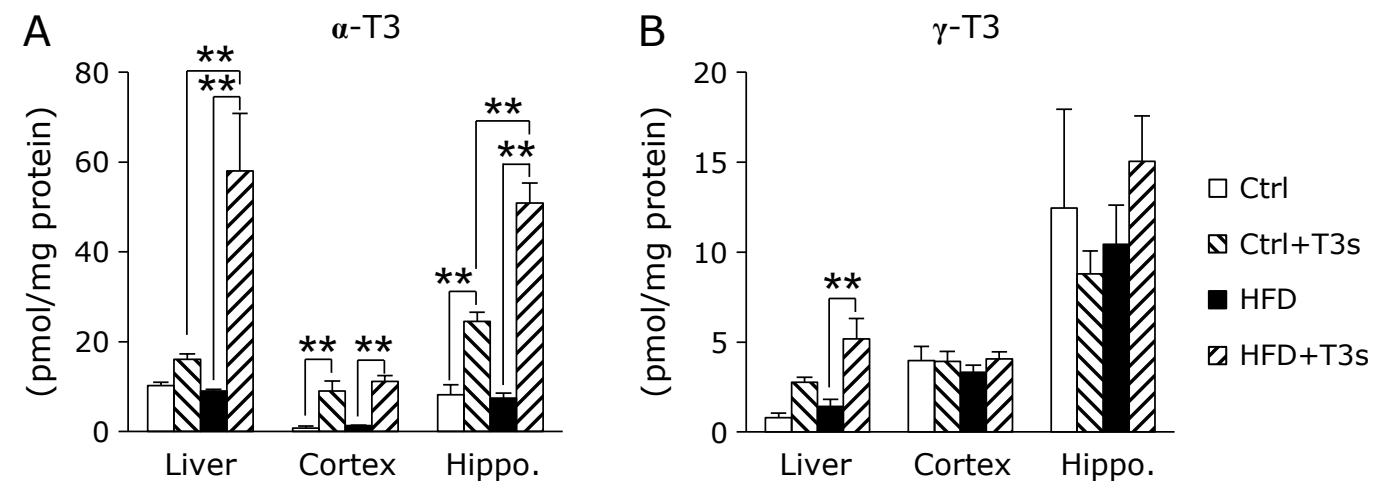

Fig. 6. $\alpha$ - (A) and $\gamma$-tocotrienol (T3) (B) levels are shown. The liver and brain tissues [cortex (Cortex), hippocampus (Hippo.)] were collected after perfusion, and $\alpha$ - and $\gamma$-T3 levels were measured using HPLC. Control (Ctrl): $n=4$, Ctrl + T3s: $n=4$, high-fat diet (HFD): $n=4$ (only Hippo.: $n=3$ ), HFD + T3s: $n=4$. The date was shown as mean \pm SE. Tukey Kramer method: $* * p<0.01$.
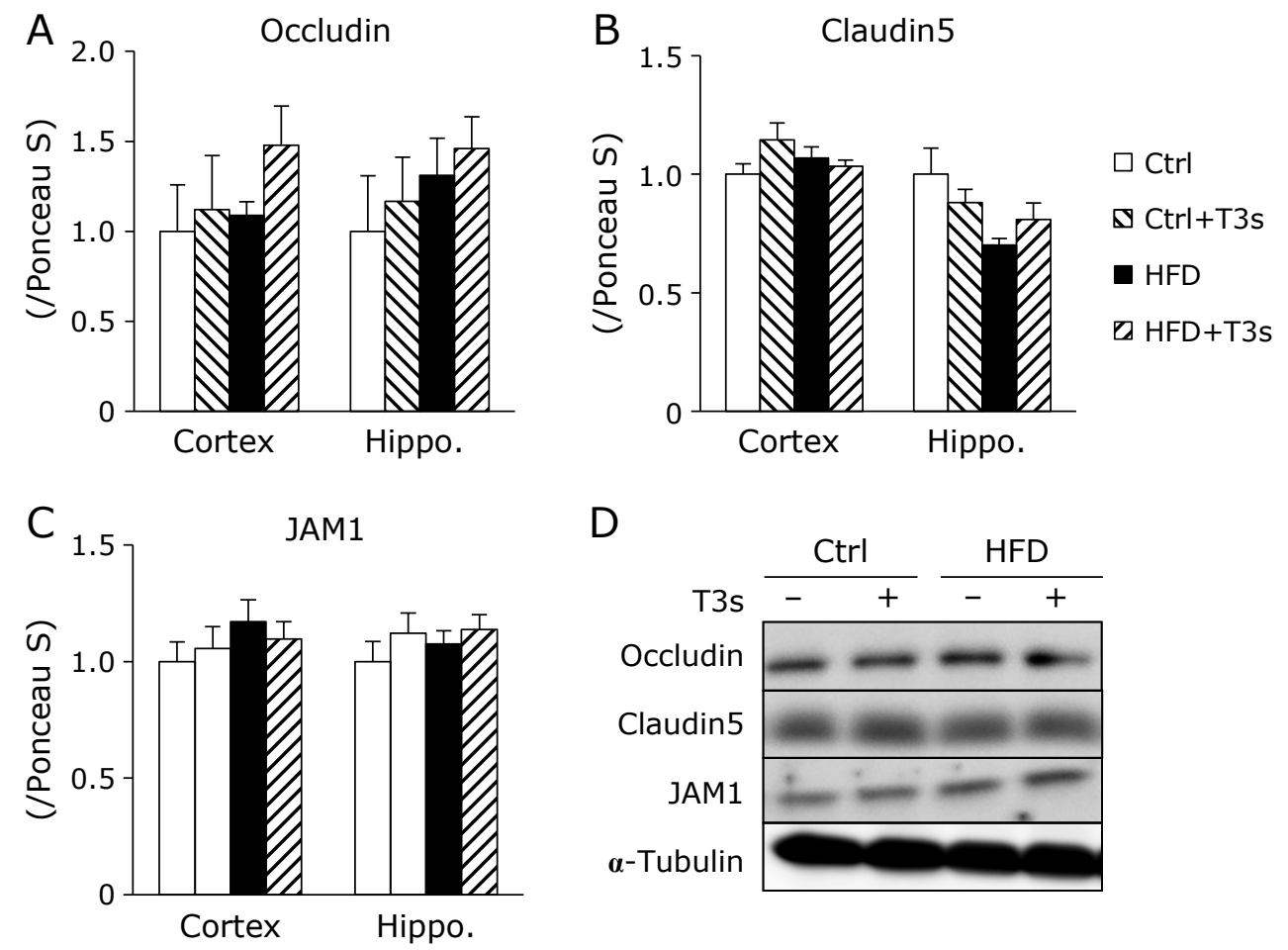

Fig. 7. BBB-related protein expressions [Occludin (A), Claudin5 (B), and JAM1 (C) and Western blotting in the cortex (D)] in cortex (Cortex) and hippocampus (Hippo.) in each group. The brain regions after perfusion were used for analysis. The relative intensities of $C$ trl in each brain region were set to 1. (D) Western blotting in the cortex. Control (Ctrl): $n=4$, Ctrl + T3s: $n=4$, high-fat diet (HFD): $n=4$, HFD + T3s: $n=4$. The date was shown as mean $\pm \mathrm{SE}$. Comparisons were performed using the Tukey Kramer method.

expression. $^{(30)}$ Jin et al. $^{(3)}$ reported that ROS induce lipid droplet accumulation in HepG2 cells. Treatment with hydrogen peroxide increased lipid droplets in HepG2 cells. On the other hand, it is well known that T3s have strong antioxidant effect in vitro and in vivo. ${ }^{(14-16)}$ From the above evidence, T3s may attenuate lipid droplet production by modulating lipid synthesis mRNA expression and/or an antioxidant effect. We are currently working on elucidating the mechanisms of these effects using in vivo and in vitro models.

Oxidative stress is significantly increased in obesity, and as a result, which may increase the risk of cognitive dysfunction. ${ }^{(4,7,8)}$ Treatment with T3s dramatically changed body weight in HFD mice in our experimental models, which indicated that T3s may also reduce the risk of oxidative damage in the brain via its antioxidant function and anti-obesity effect. However, cognitive function in the HFD group was not deteriorated compared to controls in the Morris water maze and Y-maze tests. One possible reason which is explaining these results may be the difference in diet composition. Because the Ctrl was fed the control diet against HFD, the nutrient composition of the Ctrl diet in our experimental model differed from that of a normal pellet diet (Lab MR stock; Nosan Corp., Kanagawa, Japan). The Ctrl diet may have led to over-nutrition compared to a normal pellet diet. Due to this difference, the changes in cognitive function between the Ctrl and HFD mice could not be confirmed (i.e., cognitive dysfunction may have occurred in the Ctrl mice compared to 

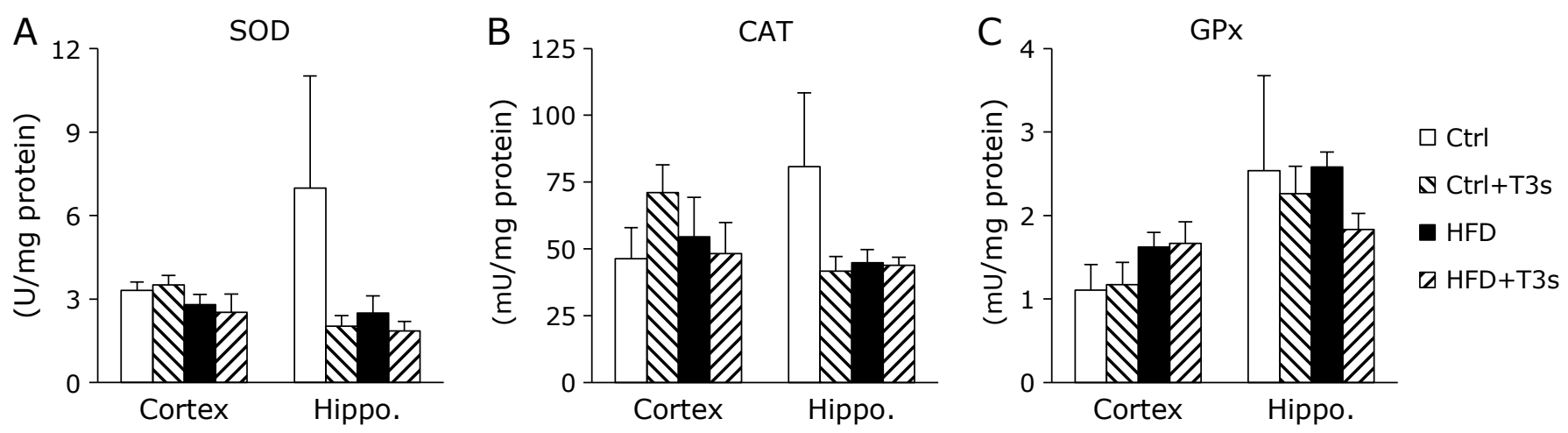

Fig. 8. The activities of antioxidative enzymes [superoxide dismutase (SOD) (A), catalase (CAT) (B), and glutathione peroxidase (GPx) (C)]. All values were measured in the cortex (Cortex) and hippocampus (Hippo.). The brain regions without perfusion were used for analysis. All enzymes in the Cortex: Ctrl: $n=6$, Ctrl + T3s: $n=6$, HFD: $n=6$, HFD + T3s: $n=6$, SOD in Hippo.: Ctrl: $n=4$, Ctrl + T3s: $n=5$, HFD: $n=5$, HFD + T3s: $n=3$, CAT and GPx in Hippo.: Ctrl: $n=5, \mathrm{Ctrl}+\mathrm{T3s}: n=6$, HFD: $n=6, \mathrm{HFD}+\mathrm{T} 3 \mathrm{~s}: n=6$. The date was shown as mean \pm SE. Comparisons were performed using Tukey Kramer method.
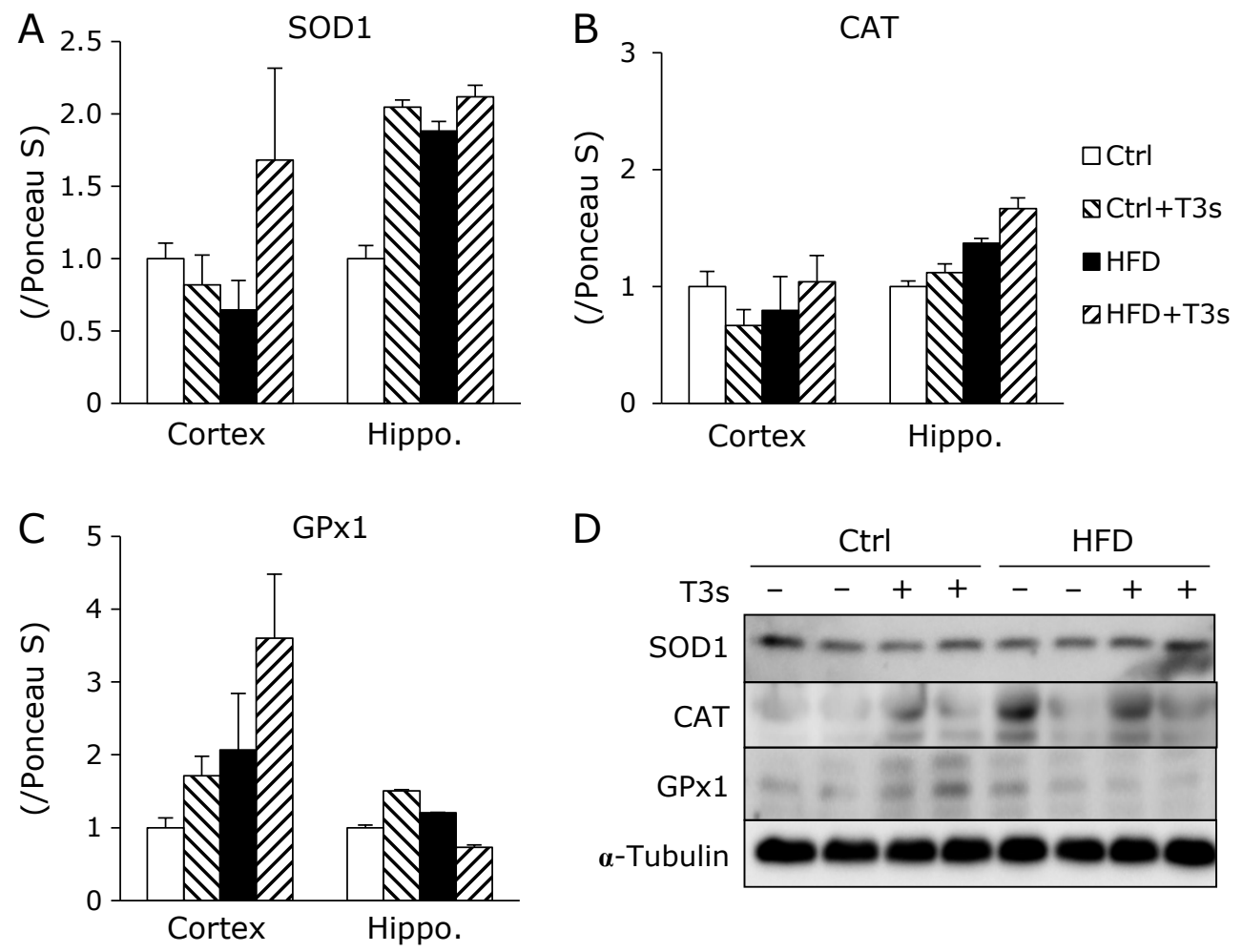

Fig. 9. Antioxidative enzyme protein expressions in the brain [superoxide dismutase (SOD)1 (A), catalase (CAT) (B), and glutathione peroxidase (GPx)1 (C) and Western blotting in the cortex (D)]. The brain regions [cortex (Cortex) and hippocampus (Hippo.)] without perfusion were used for analysis. The relative intensities of Ctrl in each brain region were set to 1. (D) Western blotting in the Cortex. Control (Ctrl): $n=6, \mathrm{Ctrl}+\mathrm{T} 3 \mathrm{~s}: n=6$, high-fat diet (HFD): $n=6$, HFD + T3s: $n=6$. The date was shown as mean \pm SE. Comparisons were performed using the Tukey Kramer method.

mice fed a normal pellet diet.). Additionally, co-treatment with Ctrl and T3s significantly improved the learning ratio in the Morris water maze test. However, no significant differences in body weight gain between $\mathrm{Ctrl}$ and $\mathrm{Ctrl}+\mathrm{T} 3 \mathrm{~s}$ were observed. From this evidence, the cognitive function improved effect of T3s might be not through anti-obesity effect. In this study, no changes were seen in antioxidative defense systems in the T3s-treated mice brains. It is well known that neurotrophic factors such as nerve growth factor (NGF) and brain derived neurotrophic factor (BDNF) are strongly related to cognitive function. ${ }^{(31,32)}$ It is possible that T3s may influence neurotrophic factor expressions. Further research is needed to clarify the mechanism underlying the improvement effect on cognitive function by T3s. Cognitive function as assessed by Rota-Rod significantly decreased in the HFD-treated mice compared to the controls in the presence or absence of T3s. A significant negative correlation was observed between body weight and the time to fall or rpm at the fall. These results indicated that Rota-Rod is not suitable for judging the cognitive function of obese mice.

We previously reported that $\mathrm{T} 3 \mathrm{~s}$ can reach the brain through oral intake. ${ }^{(21)}$ However, our samples included some blood, and 


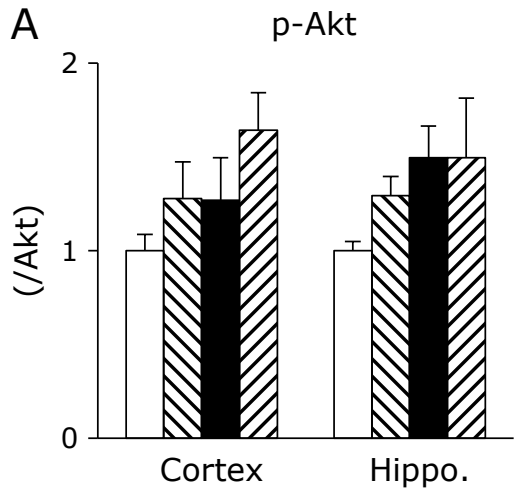

B p-mTOR
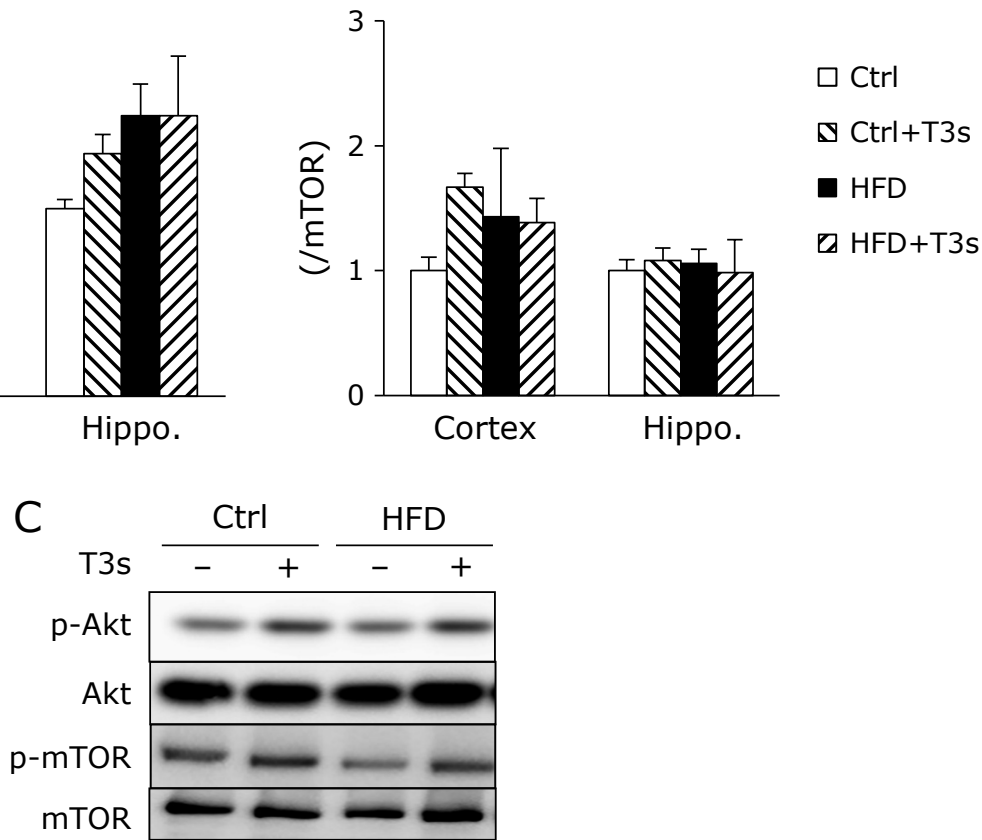

Fig. 10. Phosphorylation ratios of protein kinase $B(A k t)(A)$ and mammalian target of rapamycin (mTOR) (B). The brain regions [cortex (Cortex) and hippocampus (Hippo.)] without perfusion were used for analysis. The relative intensities of Ctrl in each brain region were set to 1. (C) Western blotting in the Cortex. Control (Ctrl): $n=6$, Ctrl + T3s: $n=6$, high-fat dirt (HFD): $n=6$, HFD + T3s: $n=6$. The date was shown as mean \pm SE. Comparisons were performed using the Tukey Kramer method.

thus, we could not judge whether T3s were from neurons or blood. To solve this problem, before measuring brain T3s levels, blood was completely removed from the tissues by perfusion. $\mathrm{T} 3 \mathrm{~s}$ could be detected in the perfused brain samples, and treatment with T3s significantly increased brain $\alpha$-T3 levels. These results indicated that $\mathrm{T} 3 \mathrm{~s}$ can certainly reach the brain through oral intake. However, there are some reports that T3s cannot through the BBB. ${ }^{(14)}$ We assessed BBB-related protein expressions using WB. We expected that $\mathrm{T} 3 \mathrm{~s}$ may reach the brain via collapsing of the BBB function. Kaneai et al. ${ }^{(14)}$ reported that treatment with T3s significantly increased brain T3s levels in normal-aged rats compared to the young controls due to decreasing BBB-related protein expressions by aging. According to our results, no significant difference was seen in BBB-related protein expressions in the brains of obese mice, which suggested that $\mathrm{T} 3 \mathrm{~s}$ may reach the brain without $\mathrm{BBB}$ dysfunction. VE is absorbed from the intestines and transported to the liver with chylomicron and chylomicron remnants. Next, VE is released from the liver with very-low-density lipoprotein to the blood and transported to tissues with low-density lipoprotein (LDL) and high-density lipoprotein (HDL). ${ }^{(33)}$ Both lipoproteins can enter the brain via each receptor. Scavenger receptor class B type 1 (SRB1) is well known as a HDL receptor, and SRB1 overexpression using adenovirus in porcine brain capillary endothelial cells (pBCECs) increases HDL-associated $\alpha$-TOC uptake. ${ }^{(34)}$ Additionally, srb $1^{-/-}$mice exhibit low $\alpha$-TOC levels in the brain. On the other hand, $\alpha$-TOC levels in the brain did not change in a model of LDL receptor knockout mice. ${ }^{(35)}$ Therefore, we think that T3s which is in HDL may be able to reach the brain; however, the detailed mechanisms remain unclear. Further study is needed to clarify the relationship between BBB function and brain $\mathrm{T} 3 \mathrm{~s}$ levels. In addition, $\alpha-\mathrm{T} 3$ levels in T3s-treated mice tissue were dramatically higher than $\gamma$-T3 levels. However, the $\gamma$-T3 composition ratio was the highest in T3s-treated diet. As we discussed in a previous study, the reason for this phenomenon may be the difference in binding affinity between $\alpha$ - or $\gamma$-T3 and $\alpha$-TOC transfer protein $\left(\alpha\right.$-TPP). ${ }^{(21)}$ Co-treatment with HFD and T3s significantly increased $\alpha$-T3 level in hippocampus compared to co-treatment with $\mathrm{Ctrl}$ and $\mathrm{T} 3 \mathrm{~s}$. As the reason of this phenomenon, we think that there is a difference of serum HDL concentration between $\mathrm{Ctrl}+\mathrm{T} 3 \mathrm{~s}$ and $\mathrm{HFD}+\mathrm{T} 3 \mathrm{~s}$ mice. Although the body weight gain of the HFD + T3s mice was significantly lower than that of the HFD mice, the body weight of the HFD + T3s mice was significantly higher than that of the $\mathrm{Ctrl}+\mathrm{T} 3 \mathrm{~s}$ mice. It is possible that serum HDL concentration of the HFD $+\mathrm{T} 3 \mathrm{~s}$ mice is higher than that of the $\mathrm{Ctrl}+\mathrm{T} 3 \mathrm{~s}$ mice. From above, hippocampal $\alpha-\mathrm{T} 3$ level in the HFD $+\mathrm{T} 3 \mathrm{~s}$ mice might be higher than that of the $\mathrm{Ctrl}+\mathrm{T} 3$ s mice.

A significant difference was found in the Morris water maze test in our experimental models, so we checked antioxidative enzyme activities and protein expressions in the brain. It is well known that brain oxidation by ROS induces cognitive dysfunction, and that ROS is scavenged by antioxidative enzymes..$^{(7,8)}$ No significant differences were seen in activities or expressions. However, treatment with T3s nominally (but not significantly) increased each activity and expression compared to the untreated groups. Additionally, Akt and/or mTOR phosphorylation was involved in lifespan extension, which confirmed under calorie restriction condition via oxidative damage attenuation. ${ }^{(26,27)}$ These proteins regulate oxidative stress via NF-E2-related factor (Nrf2) phosphorylation. ${ }^{(36,37)}$ The phosphorylation levels of Akt and mTOR were not significant difference in this study. Previously, we reported the possibility that treatment with T3s significantly increases these parameters in the brain. ${ }^{(21)}$ One reason we could not confirm the significant difference may have been the length of the treatment period. In our previous report, we set the treatment period to 5 months. On the other hand, in this study, the T3s treatment period was only 2 months. Thus, long-term treatment may be needed to modify the antioxidative defense systems in the brain by $\mathrm{T} 3 \mathrm{~s}$. 
In this study, we assessed the influences of treatment with T3s in HFD-treated mice. Treatment with T3s inhibited body weight gain and lipid droplets synthesis in the liver. Furthermore, T3s reached the brain without $\mathrm{BBB}$ dysfunction, and altered cognitive function. These findings suggest that $\mathrm{T} 3 \mathrm{~s}$ may be able to be applied as a therapeutic medicine for obesity and dementia.

\section{Author Contributions}

KF designed this study. YK performed all experiments. HU and RU performed a part of experiments. HT gave the methodology for the perfusion. YA supplied the information and knowledge about T3s. SE and SY provided the technical advices and the resources for the histological analysis. YK and KF analyzed and interpreted the data. YK wrote the draft of the article. KF reviewed and edited the article.

\section{Conflict of Interest}

No potential conflicts of interest were disclosed.

\section{References}

1 Bray GA, Heisel WE, Afshin A, et al. The science of obesity management: an endocrine society scientific statement. Endocr Rev 2018; 39: 79-132.

2 Halade GV, Kain V. Obesity and cardiometabolic defects in heart failure pathology. Compr Physiol 2017; 7: 1463-1477.

3 Jin Y, Tan Y, Chen L, Liu Y, Ren Z. Reactive oxygen species induces lipid droplet accumulation in HepG2 cells by increasing perilipin 2 expression. Int J Mol Sci 2018; 19: 3445.

4 Furukawa S, Fujita T, Shimabukuro M, et al. Increased oxidative stress in obesity and its impact on metabolic syndrome. J Clin Invest 2004; 114: 17521761.

5 Nowotny K, Jung T, Hohn A, Weber D, Grune T. Advanced glycation end products and oxidative stress in type 2 diabetes mellitus. Biomolecules 2015; 15: 194-222.

6 Yang J, Fernández-Galilea M, Martínez-Fernández L, et al. Oxidative stress and non-alcoholic fatty liver disease: effects of omega-3 fatty acid supplementation. Nutrients 2019; 11: 872

7 Fukui K, Nakamura K, Shirai M, Hirano A, Takatsu H, Urano S. Long-term vitamin E-deficient mice exhibit cognitive dysfunction via elevation of brain oxidation. J Nutr Sct Vitaminol (Tokyo) 2015; 36: 362-368.

8 Netto MB, de Oliveira Junior AN, Goldim M, et al. Oxidative stress and mitochondrial dysfunction contributes to postoperative cognitive dysfunction in elderly rats. Brain Behav Immun 2018; 73: 661-669.

9 Walker JM, Harrison FE. Shared neuropathological characteristics of obesity, type 2 diabetes and Alzheimer's disease: impacts on cognitive decline. Nutrients 2015; 7: 7332-7357.

10 Letra L, Santana I, Seiça R. Obesity as a risk factor for Alzheimer's disease: the role of adipocytokines. Metab Brain Dis 2014; 29: 563-568.

11 Wu L, Meng J, Shen Q, et al. Caffeine inhibits hypothalamic $\mathrm{A}_{1} \mathrm{R}$ to excite oxytocin neuron and ameliorate dietary obesity in mice. Nat Commun 2017; 8: 15904.

12 Mao QQ, Xu XY, Cao SY, et al. Bioactive compounds and bioactivities of ginger (Zingiber officinale Roscoe.). Foods 2019; 8: 185.

13 Neophytou CM, Constantinou AI. Drug delivery innovations for enhancing the anticancer potential of vitamin $\mathrm{E}$ isoforms and their derivatives. Biomed Res Int 2015; 2015: 584862.

14 Kaneai N, Sumitani K, Fukui K, Koike T, Takatsu H, Urano S. Tocotrienol improves learning and memory deficit of aged rats. $J$ Clin Biochem Nutr 2016; 58: 114-121.

15 Fukui K, Takatsu H, Koike T, Urano S. Hydrogen peroxide induces neurite degeneration: prevention by tocotrienols. Free Radic Res 2016; 45: 681-691.

16 Fukui K, Ushiki K, Takatsu H, Koike T, Urano S. Tocotrienols prevent hydrogen peroxide-induced axon and dendrite degeneration in cerebellar cells. Free Radic Res 2012; 46: 184-193.

17 Zhao L, Kang I, Fang X, et al. Gamma-tocotrienol attenuates high-fat dietinduced obesity and insulin resistance by inhibiting adipose inflammation and M1 macrophage recruitment. Int J Obes (Lomd) 2014; 39: 438-446.

18 Tham SY, Loh HS, Mai CW, Fu JY. Tocotrienols modulate a life or death decision in cancer. Int J Mol Sci 2019; 20: 372.

19 Wong WY, Ward LC, Fong CW, Yap WN, Brown L. Anti-inflammatory $\gamma$ and $\delta$-tocotrienols improve cardiovascular, liver and metabolic function in diet-induced obese rats. Eur J Ntur 2017; 56: 133-150.

20 Fukui K, Shirai M, Ninuma T, Kato Y. Anti-obesity effects of tocotrienols and bran in high-fat diet-treated mice. Nutrients 2019; 11: 830
21 Kato Y, Aoki Y, Fukui K. Tocotrienols influence body weight gain and brain protein expression in long-term high-fat diet-treated mice. Int J Mol Sci 2020; 21: 4533 .

22 Daneman R, Part A. The blood-brain barrier. Cold Spring Harb Perspect Biol 2020; 7: a020412.

23 Pan R, Yu K, Weatherwax T, Zheng H, Liu W, Liu KW. Blood occluding level as a potential biomarker for early blood brain barrier damage following ischemic stroke. Sci Rep 2017; 7: 40331.

24 Knowland D, Arac A, Sekiguchi K, et al. Stepwise recruitment of transcellular and paracellular pathways underlies blood-brain barrier breakdown in stroke. Neuron 2014; 82: 603-617.

25 Garrido-Urbani S, Brandfield PF, Imhof BA. Tight junction dynamics: the role of junctional adhesion molecules (JAMs). Cell Tissue Res 2014; 355: 701-715.

26 Mercken EM, Crosby SD, Lamming DW, et al. Calorie restriction in human inhibits the $\mathrm{PI} 3 \mathrm{~K} / \mathrm{AKT}$ pathway and induces a younger transcription profile. Aging Cell 2013; 12: 645-651.

27 de Cabo R, Carmona-Gutierrez D, Bernier M, Hall MN, Maeda F. The search for anti-aging interventions: from elixirs to fastingregimens. Cell 2014; 157: $1515-1526$

28 Carr RM, Ahima RS. Pathophysiology of lipid droplet proteins in liver diseases. Exp Cell Res 2016; 340: 187-192.

29 Li X, Wei X, Sun Y, et al. High-fat diet promotes experimental colitis by inducing oxidative stress in the colon. Am J Physiol Gastrointest Liver Physiol 2019; 317: G453-G462.

30 Burdeos GC, Nakagawa K, Watanabe A, Kimura F, Miyazawa T. $\gamma-$ Tocotrienol attenuates triglyceride through effect on lipogenic gene expressions in mouse hepatocellular carcinoma Hepa 1-6. J Nutr Sci Vitaminol 2013; 59: 148-151.

31 Rocco ML, Soligo M, Manni L, Aloe L. Nerve growth factor: early studies and resent clinical trials. Curr Neuropharmacol 2018; 16: 1455-1465.

32 Bekinschtein P, Cammarota M, Medina JH. BDNF and memory processing. Neuripharmacology 2013; 76 Pt C: 677-683.

33 Rigotti A. Absorption, transport, and tissue delivery of vitamin E. Mol Aspects Med 2013; 28: 423-436.

34 Goti D, Hrzenjak A, Levak-Frank S, et al. Scavenger receptor class B, type 1 is expressed in porcine brain capillary endothelial cells and contributes to selective uptake of HDL-associated vitamin E. J Neurochem 2001; 76: 498508.

35 Lee P, Ulatowski LM. Vitamin E: mechanism of transport and regulation in the CNS. IUBMB Life 2019; 71: 424-429.

36 Martin D, Rojo A, Salinas M, et al. Regulation of heme oxygenase-1 expression through the phosphatidylinositol 3-kinase/Akt pathway and the Nrf2 transcription factor in response to the antioxidant phytochemical carnosol. $J$ Biol Chem 2004; 279: 8919-8929.

37 Taguchi K, Hirano I, Itoh T, et al. Nrf2 enhances cholangiocyte expansion in pten-deficient livers. Mol Cell Biol 2014; 34: 900-913.

This is an open access article distributed under the terms of the Creative Commons Attribution-NonCommercial-NoDerivatives License (http://creativecommons.org/licenses/by-nc-nd/4.0/). 